\title{
Planetary systems formation and the diversity of extrasolar systems
}

\author{
Yamila Miguel $^{1,2}$, Octavio M. Guilera ${ }^{1,2}$, and Adrián Brunini ${ }^{1,2}$ \\ ${ }^{1}$ Facultad de Cs. Astronómicas y Geofísicas - Universidad Nacional de La Plata, Argentina \\ ${ }^{2}$ Instituto de Astrofísica de La Plata (IALP) - CONICET \\ email: ymiguel@f caglp.unlp.edu.ar
}

\begin{abstract}
With the end of answer questions as, how common are planetary systems like our own in the Universe? and What is the diversity of planetary systems that we could find in the universe?, we develop a semi-analytical model for computing planetary systems formation and consider different initial conditions for generating a large sample of planetary systems, which is analysed statistically. We explore the effects in the planetary system architecture of assuming different initial disc profiles and planetary migration rates.
\end{abstract}

Keywords. planets and satellites: formation, planetary systems: formation

\section{Introduction}

The set of planetary systems discovered orbiting around single stars in the solar neighborhood is remarkably diverse and displays a wide range of architectures that reflect the process of planetary formation and are a consecuence of the environment where they were born. Our main objective is to explore the importance of several factors in defining the architecture of a planetary system, explaining the observed diversity of planetary systems. To this end, we explore different gas and solids disc profiles, as well as different planetary migration rates, to find out which factors reproduce the different observed planetary systems and then predict the systems that will be the more common and thus those expected to be find in the Universe.

\section{A Brief Description of the Model}

Our model for planetary systems formation is based on our previous works (e.g. Miguel \& Brunini 2009; Miguel et al. 2010) and its essence is:

Following last protoplanetary discs observations (Andrews et al. 2009), we assume that the gas and solid surface density are characterized by a power-law in the inner part of the disc, with an exponent $\gamma$ which take different values (we assume $\gamma=0.5,1$ and 1.5) and an exponential decay in the outer part.

The first initial core is located at the inner edge of the disc, the rest of the cores are separated $10 R_{H}$ each other until the end of the disc.

The cores grow in the oligarchic growth regime (Ida \& Makino 1993) and they also grow due to collisions with other embryos. When a core reaches the critical mass, the gas accretion process begins, whose rate was fitted from the results of Fortier et al. (2009).

We consider type I and II planetary migration. Type I is very fast and a factor $c_{m i g I}$ is assumed for delaying it. We assume $c_{m i g I}=0.01,0.1$ and 0 , which represent a migration delayed 100, 10 times, and not considered, respectively. 
Table 1.

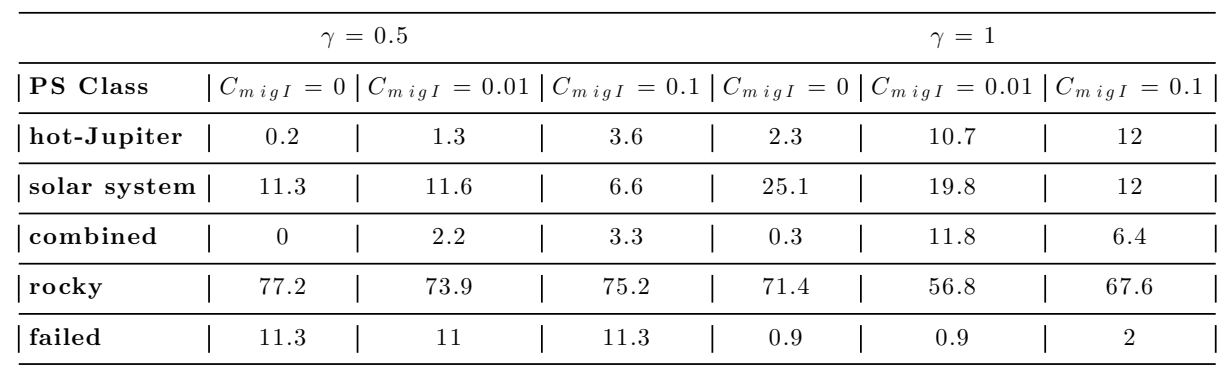

\begin{tabular}{|c|c|c|c|}
\hline \multicolumn{4}{|c|}{$\gamma=1.5$} \\
\hline PS Class & $\mid C_{m i g I}=0$ & $C_{m i g I}=0.01$ & $\left|C_{m i g I}=0.1\right|$ \\
\hline |hot-Jupiter | & 7.7 & 13.2 & 16.9 \\
\hline | solar system | & 22.6 & 20 & 9.5 \\
\hline combined & 0.8 & 11.6 & 2.2 \\
\hline rocky & 68.1 & 54.4 & 49 \\
\hline failed & 0.8 & 0.7 & 22.4 \\
\hline
\end{tabular}

\section{Results}

We perform 12 simulations, in each one we explore the 3 different gas and solids disc density profiles considered and also the different planetary migration rates. In each simulation 1000 planetary systems are formed, where the initial conditions for our discs are chosen random according to the observations.

Since most of the observed planets are giant planets, we use them for a new planetary systems classification:

hot-Jupiter systems: these planetary systems host planets with masses larger than $15 M_{\oplus}$ at a distance less than $1 \mathrm{au}$.

solar systems: these systems harbor giant planets located between 1 and $30 \mathrm{au}$.

combined systems: these systems harbor at least one giant planet within $1 \mathrm{au}$ and also in the middle part of the disc.

cold-Jupiter systems: in this case the giant planets are located further from $30 \mathrm{au}$.

rocky systems: these systems have only planets with masses less than $15 \mathrm{M}_{\oplus}$.

According to this new classification, we analyse statistically the population of planetary systems generated with our model. The table shows the $\%$ of different kinds of planetary systems found when assuming different values of $\gamma$ and different $c_{m i g I}$. We exclude the cold-Jupiter systems, since we are unable to form this systems with our model.

As seen rocky systems are the most common in the Universe. We also found that solar systems are not expected to be rare in the Universe, being their formation favoured when $\gamma=1$ and slow migration rates. Finally, the hot-Jupiter systems are born in discs with $\gamma=1.5$ and fast migration rates.

\section{References}

Andrews, S. M., et al. 2009, ApJ, 700, 1502

Fortier, A., Benvenuto, O., \& Brunini, A. 2009, A\&A, 500, 1249

Ida, S. \& Makino, J. 1993, Icarus, 106, 210

Miguel, Y. \& Brunini, A. 2009, MNRAS, 392, 391

Miguel, Y., Guilera, O. M., \& Brunini, A. 2011, MNRAS, 412, 2113 